\title{
LA REVISTA PERUANA DE MEDICINA EXPERIMENTAL Y SALUD PÚBLICA AHORA INDIZADA EN MEDLINE/INDEX MEDICUS
}

\section{[THE REVISTA PERUANA DE MEDICINA EXPERIMENTAL Y SALUD PÚBLICA NOW INDEXED IN MEDLINE/INDEX MEDICUS]}

\author{
Zuño Burstein ${ }^{1}$, Percy Mayta-Tristán ${ }^{2}$
}

La Revista Peruana de Medicina Experimental y Salud Pública (RPMESP) ha sido aceptada, a partir del año 2010, en MEDLINE/Index Medicus, la base de datos internacional de mayor cobertura e importancia en salud a nivel mundial. Desde julio de este año, la RPMESP se une a las 5497 revistas científicas que forman parte de la colección de MEDLINE, donde solo $96(1,75 \%)$ son revistas en español; es la segunda revista peruana -junto con la Revista de Gastroenterología del Perú- que dispone sus contenidos actualmente en esta base de datos.

En el año 2008, el Comité Editor de la RPMESP manifestó su interés de trabajar el ingreso, en un mediano plazo, a MEDLINE; se hizo un análisis de lo avanzado en cuanto a mejoras del proceso editorial, en particular de la revisión por pares, la cual ahora es electrónica y con un mayor número de revisores internacionales, ello repercutió en la optimización del proceso tanto en calidad como en rapidez ${ }^{(1)}$. En el año 2009, en un editorial previo ${ }^{(2)}$, se informó que existía la decisión política de la Jefatura del Instituto Nacional de Salud (INS), a cargo del Dr. Aníbal Velásquez, respaldada por el Ministro de Salud Dr. Óscar Ugarte, de brindar el apoyo para que el mencionado ingreso sea posible; para ello, se reconstituyó el Comité Editor del INS y se incluyó a un mayor número de miembros externos a la institución, se amplió el Consejo Consultivo y se incrementó el personal dedicado a la RPMESP (un editor adjunto y una secretaria administrativa). Gracias a estos cambios, a inicios del 2010 informamos que ya cumplíamos con todos los requisitos exigidos por MEDLINE y que nuestra aplicación de evaluación había sido enviada los primeros días de febrero de este año ${ }^{(3)}$.

La RPMESP inició su edición en 1942 y continuó hasta 1960 en la que lamentablemente fue interrumpida; reinició una segunda época en 1997, año desde el cual ha existido continuidad ${ }^{(1)}$. Se debe destacar, sin embargo, que el interés y apoyo a nuestra Revista se intensificó en las diferentes gestiones del INS y del MINSA desde el año 2000.

Estudios previos, ya señalaron que la RPMESP es la publicación con mayor producción de artículos en general y de investigación en particular, dentro de todas las revistas peruanas ${ }^{(4)}$; así mismo, es la que en el año 2009 tenía mayor factor de impacto medido en Scholar Google ${ }^{(5)}$; recientemente se ha demostrado que tiene citas en revistas ISI y su factor de impacto en esa base, aunque muy pequeño aún, viene en incremento (6). Adicionalmente, investigadores independientes manifestaron que la RPMESP es la más importante revista médica peruana en salud pública ${ }^{(7)}$, argumentos que fueron parte de nuestra postulación a MEDLINE.

Entre los diversos requisitos requeridos por MEDLINE, el que más retrasaba nuestra posibilidad de aplicación era la periodicidad en la publicación, problema crónico de las revistas peruanas (8); en ese sentido, Pamo demostró en el 2005 que la RPMESP tenía dos años de atraso ${ }^{(8)}$. El reto del equipo editorial fue lograr publicar la revista con puntualidad sin perder la calidad; es más, se incrementó el número de artículos de nueve en promedio el 2005, a 25 en el 2009; recién cuando se empezó a publicar oportunamente fue que se envió la aplicación a MEDLINE.

Otro aspecto relevante ha sido cambiar el carácter local o institucional de la RPMESP pues pasó a ser una revista abierta y de interés internacional. Por ejemplo, en el periodo 2002-2004 el $70 \%$ de artículos era de autores

Médico dermatólogo y tropicalista, Director de la Revista Peruana de Medicina Experimental y Salud Pública, Académico de Número de la Academia Nacional de Medicina, Profesor emérito de la Universidad Nacional Mayor de San Marcos. Lima, Perú. Correo electrónico: zburstein@ins.gob.pe

2 Médico salubrista, Editor científico de la Revista Peruana de Medicina Experimental y Salud Pública, Instituto Nacional de Salud. Lima, Perú.pmayta@ins.gob.pe 
del INS, en el 2009 pasó a ser menos del 30\% y uno de cada cuatro artículos tenía al menos un autor extranjero. Por otro lado, en el 2005 el 56\% los revisores era del INS y en el 2009 representaron solo el $8 \%$; todo ello aunado a la apertura en la conformación del Comité Editor ${ }^{(9)}$.

El recurso humano es un aspecto básico para lograr los objetivos que se plantea cualquier institución, y uno de los retos mayores ha sido que en el contexto de una entidad gubernamental, donde hay continuos cambios políticos en la dirección de la institución, se logró un consenso de que la RPMESP debería ser apoyada e impulsada, por lo que a pesar de cuatro cambios de gestión en el INS, en el periodo 2005-2009, no se reemplazó al Comité Editor-como suele suceder en otras revistas peruanas- sino que se repotenció, siempre bajo el liderazgo del Dr. Zuño Burstein y el Dr. César Cabezas, junto con los editores científicos Percy Mayta-Tristán y Edward Mezones-Holguín. Así mismo, participaron de este proceso como miembros del Comité, Pedro Álvarez-Falconí, Elizabeth Anaya Ramírez, Rosario Belleza Zamora, Walter H. Curioso, Alfredo Guillén Oneeglio, Claudio F. Lanata, J. Jaime Miranda, Sergio Recuenco Cabrera, Oswaldo Salaverry García, Lely Solari Zerpa, Alonso Soto Tarazona y Javier Vargas Herrera, cuyos créditos han sido señalados previamente ${ }^{(2)}$. Merece especial mención el apoyo administrativo otorgado con singular eficiencia e identificación con su responsabilidad, de José Villegas Ortega, Francisco Quispe Álvarez y Mónica Suárez Aguilar, a cargo de la coordinación técnico administrativa de la Revista en diferentes periodos, así como de Graciela Rengifo García, encargada de la distribución de la Revista, Carolina Tarqui Mamani, asistente editorial y Daniel Cárdenas-Rojas, corrector de estilo de la revista. Asimismo, se repotenció un Consejo Consultivo conformado por investigadores con alto nivel científico, vinculados con la Salud Pública, tanto nacionales como extranjeros.

Cuando se consideró que ya se cumplía con los requisitos para ingresar a MEDLINE, se armó el expediente para la postulación, tarea que fue realizada por el Comité Editor en su conjunto; luego dicho expediente fue revisado por investigadores de habla inglesa como David A. Moore (Imperial College London) y Roger Glass (Fogarty International Center), y editores de revistas de alto impacto como Jay Kaufman (Epidemiology), Emma Veitch (PLoS Clinical Trials) y George Davey Smith (International Journal of Epidemiology), a todos ellos nuestro agradecimiento.

La RPMESP fue evaluada en junio de 2010 por el Literature Selection Technical Review Committe de MEDLINE - quienes se reúnen solamente tres veces por año: febrero, junio y octubre - dentro de un grupo de 140 revistas; en julio comunicaron los resultados, en esta oportunidad ingresaron 39 revistas a MEDLINE, dentro de las cuales la RPMESP fue la única en español.
El reto ahora es seguir mejorando la calidad de la Revista y, en un futuro, incrementar el número de ediciones anuales puesto que cada vez recibimos un mayor número de trabajos; así por ejemplo, a finales de agosto de este año ya hemos superado el número de artículos recibidos en todo el año 2009, por lo que se tiene previsto incrementar el recurso humano dedicado a la Revista, todo esto con el fin de que la RPMESP pueda ingresar pronto a Science Citation Index del Information Scientific Institute (ISI) y formar parte del selecto grupo de revistas de todas las ciencias que congregan la información más relevante en el mundo, este aspecto es trascendental no solo para la revista sino para los indicadores científicos del Perú, ya que si la RPMESP ingresa, se incrementaría en al menos $40 \%$, la cantidad de artículos peruanos de medicina en ISI (10).

En ese contexto, es muy importante el número que ahora presentamos, pues tiene como tema central la "Política de Investigación en Salud", en él se abordan los planteamientos generales e información específica, sobre uno de los asuntos de mayor importancia e interés para el desarrollo del país, como es la investigación científica y la publicación de sus resultados que permitan hacer un diagnóstico situacional y proyecciones futuras, así como la necesidad de construir el Sistema Nacional de Investigación en Salud, como un proceso incluyente, que contribuya con evidencias a la solución de los problemas de salud del país. Para el desarrollo de este tema se ha convocado a personalidades extranjeras y nacionales, actores en los diferentes niveles y responsabilidades en tareas de investigación médico científica, para que colaboren mediante artículos originales, temas de revisión, artículos de opinión para el simposio y cartas al editor. Se ha invitado a investigadores de diferentes instituciones nacionales como el Instituto Nacional de Salud, la Dirección General de Epidemiología (MINSA), la Universidad Peruana Cayetano Heredia, la Universidad Nacional Mayor de San Marcos, el Consejo Nacional de Ciencia y Tecnología (CONCYTEC) y la Academia Nacional de Ciencias; también a instituciones internacionales como la Harvard University, la Fundación Oswaldo Cruz, la Universidad Central de Venezuela, el CONICYT-Chile, la Fundación Ford y la Organización Panamericana de la Salud.

Con lo publicado en este número queda abierto el debate sobre este importante problema, que es de particular interés para el INS, brazo científico del Ministerio de Salud del Perú, así como para las instituciones con responsabilidad en la investigación médico-científica en el país.

Bajo este marco de referencia y con el decidido afán de lograr la tan anhelada integración latinoamericana, por lo menos en aspectos relacionados con la investigación 
científica en salud pública y su difusión entre nuestros países hermanos, reiteramos nuestra política editorial de poner a disposición nuestra publicación para que los investigadores puedan enviar sus artículos a la Revista Peruana de Medicina Experimental y Salud Pública ajustándose a nuestras instrucciones para los autores.

\section{REFERENCIAS BIBLIOGRÁFICAS}

1. Cabezas C, Mayta-Tristán P. Evolución y perspectivas de la Revista Peruana de Medicina Experimental y Salud Pública, 1945-2008. Rev Peru Med Exp Salud Publica. 2008;25(2)167-68.

2. Burstein Z. La Revista Peruana de Medicina Experimental y Salud Publica en el año 2009. Rev Peru Med Exp Salud Publica. 2009;26(1):3-4.

3. Burstein Z. Cabezas C. Avances y retos de la Revista Peruana de Medicina Experimental y Salud Publica al 2010. Rev Peru Med Exp Salud Publica. 2010;27(1):3-5.
4. Huamaní C, Pacheco-Romero J. Visibilidad y producción de las revistas biomédicas peruanas. Rev Gastroenterol Peru. 2009;29(2):132-39.

5. Huamaní C, Mayta-Tristán P. Factor de impacto modificado de las revistas médicas indizadas en SciELO Perú. Rev Peru Med Exp Salud Publica. 2008;25(3):349-50.

6. Huamaní $\mathbf{C}$. Análisis de la producción, visibilidad y citación de la Revista Peruana de Medicina Experimental y Salud Pública, 2002-2009. Rev Peru Med Exp Salud Publica. 2010;27(3):367-72.

7. Cáceres CF, Mendoza W. Globalized research and "national science": the case of Peru. Am J Public Health. 2009;99(10):1792-98.

8. Pamo O. Estado actual de las publicaciones periódicas científicas médicas del Perú. Rev Med Hered. 2004;16(1):65-73.

9. [No author listed]. Revisores del año 2009. Rev Peru Med Exp Salud Publica. 2009;26(4):591-94.

10. Huamani C, Mayta-Tristán P. Producción científica peruana en medicina y redes de colaboración, análisis del Science Citation Index 2000-2009. Rev Peru Med Exp Salud Publica. 2010;27(3):315-25.

En el momento de estar redactando este editorial, hemos recibido la noticia del sensible fallecimiento del Dr. Germán Battistini More, ex Director General del Instituto Nacional de Salud, Presidente en ejercicio de la Asociación de Médicos Cesantes del Ministerio de Salud y miembro del Comité Consultivo de la RPMESP, acaecido el 30 de agosto de 2010, en Lima. Su vida se extinguió a los 93 años, después de una larga afección con prolongada postración física pero con conservación íntegra de su intelecto hasta el último momento de vida, la que dedicó en forma casi exclusiva al servicio de la salud pública peruana. Fue el Dr. Battistini, junto con el Dr. Oscar Miró Quesada Cantuarias, uno de los más destacados Directores de esta institución, la que alcanzó durante estas gestiones el más alto nivel de desarrollo, siguiendo el camino iniciado por el Dr. Telémaco Battistini su primer Director y fundador conjuntamente con el Dr. Carlos Gutiérrez Noriega, de esta Revista. El Dr. Telémaco Battistini estuvo ligado familiarmente al Dr. Germán Battistini More, cuya desaparición lamentamos y expresamos públicamente nuestro agradecimiento institucional y homenaje póstumo. 